Article

\title{
Are limits on cosmic rotation from analyses of the cosmic microwave background credible?
}

Michael J. Longo

Department of Physics, University of Michigan, Ann Arbor, MI 48109, USA; email: mlongo@umich.edu

\begin{abstract}
Recent analyses of cosmic microwave background (CMB) maps have been interpreted as demonstrating that the Universe was born without an initial rotation. However, these analyses are based on unrealistic models and do not contain essential ingredients such as quantum effects, the strong, weak and gravitational interactions between the components, their intrinsic spins and magnetic moments, as well as primordial black holes. If the Universe was born spinning these effects would distribute the initial spin angular momentum among its components long before the $\mathrm{CMB}$ forms at recombination. A primordial spin would now appear as a nonzero total angular momentum of its components along the direction of the original spin, and a primordial large-scale rotation would no longer be apparent. The existence of a special axis or direction would break a fundamental symmetry assumed in general relativity, cosmic isotropy, and a net angular momentum implies a cosmic parity violation.
\end{abstract}

Keywords: Cosmic anisotropy; cosmic microwave background; cosmic rotation; Big Bang spin; parity violation; cosmic axis

\section{Introduction}

An important question in cosmology is the possibility that the Universe was born with a primordial spin angular momentum (PSAM). This would strongly affect its evolution during inflation as it expanded from the initial singularity to its present state. A primordial spin could also help explain some outstanding problems in cosmology as discussed below.

An article by Saadeh et al. [1] has placed apparently stringent limits on the overall rotation of the Universe at the present epoch, $\omega<3.6 \times 10^{-12} \mathrm{rad} / \mathrm{Gyr}$, based on the apparent absence of the large-scale spiral-like patterns that would be expected in the cosmic microwave background (CMB) temperature and polarization maps if there were a universal rotation. Other analyses looked for a large-scale spiral topology in the CMB [2-7].

\section{Background}

In the standard "Big Bang" cosmology the Universe originated about 13.8 Gyr ago from an initial singularity. Cosmic inflation then expanded space by a factor $\sim 10^{26}$ over a time $\sim 10^{-33}$ seconds and the Universe cooled from $\sim 10^{27} \mathrm{~K}$ down to $\sim 10^{22}$. The currently understood laws of physics general relativity, quantum chromodynamics, quantum mechanics - fail completely in the first picosecond. At $\sim 10^{-12} \mathrm{~s}$ the quark epoch began, and at $\sim 1 \mathrm{~s}$ neutrinos decoupled. Primordial black holes formed at about one second of cosmic time. Quarks and gluons decoupled and via strong interactions formed hadrons. Nucleosynthesis began at $\sim 2$ minutes. The CMB did not appear until about 370,000 years when the universe finally cooled enough for neutral atoms to form 
("recombination") [8]. After recombination photons could propagate freely and diffusion damping reduced anisotropies, making the Universe itself and the cosmic microwave background radiation more uniform [9].

Magnetic fields would play a critical role in this evolution. The chaotically moving electrical charges generate magnetic fields. The quark-scale magnetic fields resulting from the quark magnetic moments would grow in scale when they condense from the quark-gluon plasma and form hadrons that in turn form nuclei. Since the quark/hadron magnetic moments are coupled to their spin angular momenta their spins would preferentially align both with the local magnetic field and with the axis of a PSAM, much like a ferrimagnetic material in a magnetic field. These primordial magnetic fields would not be affected by diffusion damping $[10,11]$ and would persist through the expansion and into the post-recombination era.

\section{Discussion}

Saadeh et al. placed apparently stringent limits on the overall rotation of the Universe based on the apparent absence of the large-scale spiral-like patterns that would be expected in the CMB temperature and polarization maps if there were a universal rotation [1]. Other analyses looked for a large-scale spiral topology in the CMB [2-7]. None of these analyses would have been sensitive to a PSAM that had already been transferred to smaller scales before the CMB formed at recombination. The appearance of the expected characteristic patterns comes from models that treat the energy content of the rotating universe before the $\mathrm{CMB}$ forms as a sum of perfect nonturbulent fluids consisting of a baryon-photon plasma and dark matter, each with their own peculiar velocity [1]. These simple models are unrealistic and do not contain essential ingredients such as quantum effects, the strong, weak and gravitational interactions between the components, as well as their intrinsic spins and the magnetic fields their chaotic motions generate.

These default models assume that the Universe was homogeneous even on microscopic scales. A persistent fragmentation on any distance scale would invalidate the assumption of a smooth adiabatic flow. The formation of quarks, leptons, nuclei, etc. with their intrinsic magnetic fields guarantees fragmentation and a PSAM would help maintain its growth. Another source of fragmentation on still larger scales is the appearance of primordial black holes during the QCD phase transition. (See, e.g., Sobrinho, Augusto, \& Gonçalves [12] and references therein.)

A quantum mechanical model of the evolution that includes turbulence suggests that even without an initial spin, seed fragmentation already occurred at around 300 years [13].

If a PSAM was already transferred to smaller scales before recombination it would not cause a significant large-scale imprint on the CMB. This same criticism would apply to any tests for a PSAM based on the CMB. It may be that the only observable evidence now for a primordial spin is a parity-violating axis ${ }^{\dagger}$ along the direction of the initial spin. There is evidence for such an axis appearing as a preferred spin orientation for spiral galaxies [14]. In addition, there is an extensive

\footnotetext{
${ }^{+}$I use axis here to refer to a particular direction in a homogeneous space that is not tied to a specific point.
} 
literature on possible evidence for preferred axes in the $\mathrm{CMB}$ and supernova distributions though none of these are explicitly related to spin. (See, for example, $[4,5,6]$.)

A primordial spin would also help to explain some outstanding problems in cosmology:

(i) Despite their ubiquity an understanding of the origin of cosmic magnetic fields remains controversial. Various scenarios have been proposed for their existence including primordial fields generated by currents formed at the electroweak or at the QCD phase transition [15]. A difficulty that arises in these scenarios is how to propagate these fields from the quark-hadron scale to much larger scales. This growth of the primordial magnetic fields would be facilitated by a PSAM. The lepton-quark-gluon-hadron magnetic moments are aligned with their spin angular momenta. As the quarks fuse into hadrons their spins must realign, and their spins and magnetic moments will preferentially align with the axis of the PSAM. The PSAM breaks the symmetry and helps protect the fields against decay from diffusion and turbulence, thus allowing the fields to survive and grow in scale. In the process the PSAM is distributed throughout the Universe. Conservation of angular momentum would guarantee that the components of the present Universe would carry the original vector angular momentum along the axis of the PSAM. (Though angular momentum in the semi-classical sense is not necessarily conserved in general relativity it is generally assumed to be conserved in the actual Universe [16].)

(ii) Jedamzik and Pogosian [11] argue that the Hubble tension (see, e.g., di Valentino et al.[17] ), as well as the tension between the values of the amplitude of galaxy clustering $S_{8}$ and the matter fraction $\Omega_{m}$ in the Planck best-fit model and those inferred from the latest surveys of large-scale structure can be greatly alleviated by the small-scale, non-linear inhomogeneities in the baryon density before recombination.

(iii) Long ago, Gamow pointed out that it is surprising that rotation is so commonplace in planets, stars, and galaxies [18]. How a universe that was born without spin could evolve into our present Universe in which everything is spinning is a mystery. On the other hand, a universe that had been born spinning would readily explain why rotation is ubiquitous in our present Universe.

(iv) It is well known that in the present Universe invariance under mirror symmetry or parity is badly violated, particularly in the weak interactions. The proto-universe without spin would have no apparent mechanism to initiate a parity violation as it expanded. An initial overall PSAM violates parity. This broken symmetry would help to rationalize the appearance of parity violation in the weak interactions as the Universe expanded and cooled. Such a mechanism could prove to be an essential ingredient in a comprehensive theory of particle interactions in the early universe.

\section{Conclusions}

A realistic detailed treatment of the evolution of the Universe before recombination is not possible at this time. The individual leptons, gluons, quarks, etc. each carry their own intrinsic magnetic fields that are coupled to their spin angular momenta. The strong interactions between the gluons and quarks would cause their spins and magnetic moments to realign as they consolidate into hadrons. If the Universe were initially rotating, these interactions would cause a chaotic 
mixing of spin angular momenta with a preference for the local angular momenta to align with a PSAM. In this evolution a primordial angular momentum would be efficiently transferred from the universal to the galactic scales.

This pre-recombination picture is nothing like the smooth adiabatic expansion discussed in Saadeh et al. [1]. In the chaotic evolution before recombination, the spins of the components would preferentially realign with the initial angular momentum direction so that it is transferred from a universal rotation to a preferred rotation direction of its components. This would imply that the present Universe does not have a significant overall rotation but has a preferred direction and retains the initial overall vector angular momentum. The large-scale initial rotation would be dissipated before recombination takes place, and its imprint on the $\mathrm{CMB}$, both in temperature and polarization maps, would be completely washed out in the long interval between lepton and gluon formation and recombination $(z \sim 1000)$. All that would remain now would be a small deviation from isotropy with a preference for the spins of its component galaxies, black holes, etc. to lie along the original axis of rotation. The existence of a special axis or direction would break a fundamental symmetry assumed in general relativity, cosmic isotropy.

While analyses based on these unrealistic models [1-7] may prove that the Universe is not rotating like a wagon wheel, we cannot dismiss the possibility that the Universe has a net angular momentum now subtly distributed among its components. Unfortunately, the limits inferred from these unrealistic models have pretty much shut down considerations of a universe that was born with spin and still retains its original angular momentum.

This research received support from the University of Michigan.

The authors declare no conflict of interest.

\section{References}

1. Saadeh, D.; Feeney, S.M.; Pontzen, A.; Peiris, H.V.; McEwen, J.D. How isotropic is the Universe? Phys. Rev. Lett. 2016, 117, 131302.

2. Ade, P.A.R.; Aghanim, N.; Arnaud, M.; Ashdown, M.; Aumont, J.; Baccigalupi,C.; Banday, A.J.; R. B. Barreiro, R.B.; Bartolo,N.; Basak, S.; Battaner, E.; et al. Planck 2015 results XVIII. Astron. Astrophys. 594, 2016, A18.

3. Ade, P.A.R.; Aghanim, N.; Armitage-Caplan, C.; Arnaud, M.; Ashdown, M.; Atrio-Barandela, F.; Aumont, J.; Baccigalupi, C.; Banday, A.J.; Barreiro, R.B.; et al. Planck 2013 results. XXIII. Isotropy and statistics of the CMB. Astron. Astrophys 2014, 571, A23.

4. Copi, C.J.; Huterer, D.; Starkman, G.D. Large-scale alignments from WMAP and Planck. Mon. Not. R. Astron. Soc. 2015, 449, 3458.

5. Schwarz, D.J.; Copi, C.J.; Huterer, D.; Starkman, G.D. CMB anomalies after Planck, Class. Quantum Grav. 2016, 33, 18400 (2016). 
6. T Jaffe, T.R.; Banday, A.J.; Eriksen, H.K.; Gorski, K.M.; Hansen, F.K. Evidence of vorticity and shear at large angular scales in the WMAP data: a violation of cosmological isotropy? Astrophys. J. 2005, 629, L1.

7. McEwen, J.D.; Josset, T.; Feeney, S.M.; Peiris, H.V., Lasenby,A.N. Bayesian analysis of anisotropic cosmologies: Bianchi VIIh and WMAP. Mon. Not. R. Astron. Soc. 2013, 436, 3680.

8. Tanabashi, M. et al. (Particle Data Group), Review of Particle Physics. Phys. Rev. D 2018, 98, 030001.

9. Silk, J., Fluctuations in the primordial fireball. Nature 1967, 215, 1155;

Silk, J., Cosmic black-body radiation and galaxy formation. Astrophys. J. 1968, 151, 459.

10. Subramanian, K.; Barrow, J., Magnetohydrodynamics in the early universe and the damping of nonlinear Alfvén waves. Phys. Rev. D 1998, 58, 083502.

11. Jedamzik, K.; Pogosian, L. Relieving the Hubble tension with primordial magnetic fields. Phys. Rev. Lett. 2020,125, 181302.

12. Sobrinho, J.; Augusto, P.; Goncalves, A. New thresholds for primordial black hole formation during the QCD phase transition. Mon. Not. R. Astron. Soc. 2016, 463, 2348.

13. Gibson, C. H. The first turbulence and first fossil turbulence. Turbulence and Combustion 2004, $72,161$.

14. Longo, M.J. Detection of a dipole in the handedness of spiral galaxies with redshifts $\mathrm{z} \sim 0.04$. Phys. Lett. B 2011, 699, 224.

15. See, e.g., a recent review by T. Vachaspati, Progress on cosmological magnetic fields. arXiv: 2010.10525 (20 Oct 2020).

16. Weinberg, S., Gravitation and Cosmology (J. Wiley and Sons, New York, 1972).

17. Di Valentino, E.; Melchiorri, A.; Silk, J., Planck evidence for a closed Universe and a possible crisis for cosmology. Nat. Astron. 2020, 4, 196.

18. Gamow, G., Rotating Universe? Nature 1946, 158, 549. 